\title{
A Review on Home Security Embedded Systems Using IoT
}

\author{
Rituparna Ghosh ${ }^{1}$, R. V. Patil ${ }^{2}$, M. N. Patil ${ }^{3}$, H. V. Dhande ${ }^{4}$, D. P. Marathe ${ }^{5}$ \\ ${ }^{I} P G$ student (M.Tech. VLSI \& Embedded Systems, ${ }^{2,3,4,5}$ Faculty E\&TC Engg. Dept \\ E\&TC Engg dept, GF's Godavari College of Engg., Jalgaon-425001
}

Received on: 16 July,2021

Revised on: 11 August, 2021

Published on: 13 August, 2021

\begin{abstract}
Internet of Things (IoT) is used to create the notion of remotely connecting and monitoring actual world objects (things) via the Internet. When it comes to our house, this idea can be aptly included to make it smarter, safer and automated. This IoT venture focuses on building a smart wireless home security machine which sends signals to the owner by the use of Internet in case of any trespass and raises an alarm optionally. Besides, the equal can also be utilized for home automation with the aid of making use of the equal set of sensors. The advantage obtained via preferring this system over the similar form of present structures is that the indicators and the repute despatched by means of the Wi-Fi connected microcontroller managed system can be acquired by using the consumer on his telephone from any distance irrespective of whether or not his mobile smartphone is related to the internet.

IoT refers to the infrastructure of connected physical gadgets which is growing at a speedy charge as massive range of units and objects are getting associated to the Internet. Home security is a very beneficial software of IoT and we are the use of it to create an less expensive security gadget for properties as well as industrial use. The device will inform the proprietor about any unauthorized entry or each time the door is opened by sending a notification to the user. After the consumer gets the notification, he can take the vital actions. The security gadget will use a microcontroller recognised as Arduino Uno to interface between the components, a magnetic Reed sensor to
\end{abstract}

monitor the status, a buzzer for sounding the alarm, and a WiFi module, ESP8266 to join and speak the usage of the Internet. The main benefits of such a gadget includes the ease of placing up, decrease charges and low maintenance.

Keywords- Home Security, IoT, Arduino, ESP8266, Smart Wireless

\section{I - INTRODUCTION}

W ireless Home security and Home automation are the twin components of this project. The presently constructed prototype of the gadget sends alerts to the proprietor over voice calls using the Internet if any sort of human motion is sensed near the entrance of his residence and raises an alarm optionally upon the user's discretion. The provision for sending alert messages to involved security personnel in case of essential scenario is also built into the system. On the different hand if the owner identifies that the person getting into his residence is no longer an intruder but an unexpected visitor of his then as a substitute of triggering the safety alarm, the user/owner can make preparations such as opening the door, switching on a range of appliances internal the house, which are also related and controlled by means of the micro-controller in the machine to welcome his guest. The same can be performed when the person 


\section{International Journal of Innovations in Engineering and Science, www.ijies.net}

himself enters the room and by means of virtue of the gadget he can make preparations from his doorstep such that as quickly as he enters his house he can make himself at full remedy except manually having to switch on the electrical appliances or his favourite T.V. channel for an example. Thus using the equal set of sensors the dual issues of domestic security and domestic automation can be solved on a complementary basis.

The indicators and the repute of the IoT machine can be accessed with the aid of the user from somewhere even the place Internet connectivity may not be readily handy (since it is not imperative for the cell cellphone to be related to web only board is required to have an access to $\mathrm{Wi}-\mathrm{Fi}$ ). The existing infra-red (IR) or Blue-tooth far flung controls existing in the market are in commonplace equipment specific and the equal can't be used interchangeably. Electrical appliances related via Bluetooth making use of Blue-tooth enabled smart telephones cannot be managed from a far-off region. Thus features such as being able to flip on an air-conditioner whilst returning home can't be carried out with such systems. In contrast, This work gives a cost fantastic and easy answer for wireless domestic automation and home protection structures. The difficulty faced through current domestic security/surveillance structures in presenting data pertaining to the state of affairs to customers whilst being away from domestic is tried to overcome in this project. IoT or Internet Things refers to the network of linked physical objects that can speak and alternate facts amongst themselves barring the want of any human intervention. It has been formally described as an "Infrastructure of Information Society", because IoT allows us to acquire information from all form of mediums such as humans, animals, vehicles, kitchen appliances. Thus any object in the physical world which can be provided with an IP address to allow records transmission over a network can be made phase of IoT system with the aid of embedding them with digital hardware such as sensors, software and networking gear. IoT is one of a kind than Internet as in a way it transcends Internet connectivity by using enabling everyday objects that uses embedded circuits to have interaction and talk with each different using the contemporary Internet infrastructure.

An vital factor to think about when we speak about home automation is Security. Home security is a very important feature of home automation and perhaps the most vital one. Home protection made a drastic changes in the previous few a long time and proceed to develop a great deal extra in the coming years. Previously home safety systems intended having an alarm that would go off when any person would break in however a clever impenetrable domestic can do a great deal extra than that. Therefore the foremost goal of our work is to diagram a machine which can alert the proprietor and others of an intruder break-in via sending a notification to their clever phones. The proprietor will also have the capacity to end or start the alarm remotely the use of just his clever phone. This system will help the users to protect their residences by way of putting the machine on the doorways or windows and monitoring the exercise through their clever phones.

There has been an remarkable increase in the quantity of gadgets being connected to the Internet in view that past few years. All these gadgets linked to the internet are part of the IoT infrastructure which can that allows these units to ship and acquire records amongst every other. This is why it is advisable to use such an existing infrastructure for designing the proposed safety system. An alarm machine that sounds the buzzer is of no use when a user is now not present in the domestic to take action. When the owner is away talk with each other. The IoT community consists of embedded electronics, sensors and software from their home, they prefer to be guaranteed that their domestic is included with the aid of intruders and thieves whilst they are gone. This is why the proposed system continues the owner knowledgeable in the actual time about the protection reputation of their home. The designed system informs the user as there is a break-in so that the consumer can take critical actions.

\section{II - LITERATURE REVIEW}

Design and Implementation of Security for Smart Home based on GSM technology was discussed by Govinda et al. (2014) that provides two methods to implement home security using IoT [1]. One is using web cameras such that whenever there is any motion detected by the camera, it sounds an alarm and sends a mail to the owner. This method of detecting intrusion is quite good, albeit somewhat expensive due to the cost of the cameras involved in the process. The cameras need to be of good quality which means it should have a wide range and the picture quality should be high enough to detect movement. Also if you go for movable cameras such as dome cameras they will cost even more than the fixed ones.

SMS based system using GSM was proposed by Karri and Daniel (2005) propose to use internet services to send messages or alert to the house owner instead of the conventional SMS.[2] Jayashri and Arvind (2013) have implemented a fingerprint based authentication system to unlock a door [3]. This system helps users by only allowing the users whose fingerprint are authorized by the owner of the house. This system can also be used to monitor who all 


\section{International Journal of Innovations in Engineering and Science, www.ijies.net}

have used the sensor to gained entry into the house. The system is coupled with a few more home protection features such as gas leakage and fire accidents. Although a good system, fingerprint sensors are expensive and complex (as they need increased sensor resolution) to integrate into an IoT setup. Some experts also argue that only relying on a fingerprint sensor is not wise as it is relatively easy to lift someone's fingerprints and replicate them, which is why it is always advised to use fingerprint scanners in a two factor authentication systems where an additional layer of security is available in the form of PIN, passcode, voice recognition, etc.

Some researchers proposed an idea of robust IoT home security system where a fault in of one component in the system does not lead to the failure of the whole system [4]. The idea of using multiple devices which may or may not be directly compatible with each other but can be made to work in such a way that they can replace an existing component of the system in case of a fault. In tandem to this, the model has the ability to use overlap between various devices which would result in preserving energy thus making the model more efficient. An example provided of the said model would use temperature sensor, Wi-Fi module and a door sensor to replace a faulty camera. The authors are successful in an effort to demonstrate the given example. However such systems are useful for people with energy efficiency in mind and for those who need a high degree of robustness with their security systems and are willing to expend more money than usual.

Laser rays and LDR sensor are used to to detect intrusion using their movement was proposed in 2016 [5]. The way the system works is that a laser is focused towards a LDR sensor and the moment that the contact of laser to LDR sensor breaks, the alarm connected to the sensor goes off alerting the neighbours and sends a SMS to the owner. This system solves the problem of covering the places which are out of range from the fixed cameras but faces the same difficulties which are faced with systems consisting of GSM modules to send text messages, which is that the delivery of message is dependent on network coverage. Also due to the nature of lasers being a straight beam, it can be avoided by intruders who know about the system and are capable of dodging the lasers, rendering the whole system useless.

A novel way to design an electronic lock using Morse code and IoT technology [6]. The authors claim that this as an original idea which have not been tried before and is the first of its kind "optical Morse code-based electronic locking system". This system uses LED"s (Light emitting diodes) as an encrypting medium to send signals. To make it more accessible to general public, the LED in smart phones has been used. On the receiver's side is a photosensitive resistor as well as a microcontroller such as Arduino processor which has the ability to decrypt the optical signal after receiving them from the LED. Upon decoding the signal it can than upload the current condition of the lock to a cloud from where the owner can monitor the system. The authors have experimented the system in real time and it has proved to work under different illumination environments with all the functions working as they were intended to. The authors also claim to have an easy and user-friendly interface. The IoT system developed here works very well and can be used by anyone and is very convenient due to the use of mobile phones as LED, which also makes it a cost expensive alternative [7]. Anitha et al (2016) proposed an home automation system using artificial intelligence and also proposed a model for cyber security systems [8].

\section{III - SYSTEM OVERVIEW}

The following systems can be used to implement Home Security Embedded Systems using IoT -

\section{1) Raspberry Pi -}

Internet of Things (IoT) conceptualizes the idea of remotely connecting and monitoring real world objects (things) through the internet .When it comes to our house, this concept can be aptly incorporated to make it smarter, safer and automated. This project presents the overall design of Home Automation System (HAS) with low cost and wireless system. The smart home concept in the system improves the standard living at home. Whenever a person come in front of the house the PIR motion detector sensor detect the person and a message will send to the house owner and he can see the person through the camera in front of the house. The camera is accessed to the webpage and also the light, fan, door lock, alarm can be controlled with the webpage. The system intended to control electrical appliances and devices in house with relatively low cost design, user-friendly interface and ease of installation. According to the priority of the person the owner can control the electrical appliances. Those who get the authorization can unlock the door by entering password on the keypad and use the home appliances depending on the priority set by the owner.

In designing a home security and automation system one or more platforms are used in order to build a reliable and flexible system that can be easily operated and adapted as a security system. Therefore, for the purpose of this project some specific deliberate choices were made on the type of platforms, hardware components and mode of operation of the home security system. 


\section{International Journal of Innovations in Engineering and Science, www.ijies.net}

\section{A: PRELIMINARY CONSIDERATIONS}

Before the actual design of the project work, specific deliberate choices in selection of appropriate implementation platforms and hardware components were made. Priority was given to low cost availability, reliability, flexibility and simplicity in all these selections.

\section{B: SYSTEM DESCRIPTION}

The PIR sensor can detect the presence of human being who enter into the house. The Raspberry Pi camera module can capture the images and live streaming videos. The owner can directly login and interact with the webpage and the control unit provided. The images captured and the videos recorded will be directly streamed on user pre-decided webpage on smart phone. User can access the video directly using the static IP address or can also stream on local domain with the help of websites.

Raspberry Pi

Raspberry Pi board is a miniature marvel, packing considerable computing power into a footprint no larger than a credit card. The processor at the heart of the Raspberry Pi system is a Broadcom BCM2835 system-onchip (SoC) multimedia processor. This means that the vast majority of the system's components, including its central and graphics processing units along with the audio and communications hardware, are built onto that single component hidden beneath the $512 \mathrm{MB}$ memory chip at the centre of the board. It's not just this SoC design that makes the BCM2835 different to the processor found in your desktop or laptop, however. It also uses a different instruction set architecture (ISA), known as ARM. The Raspberry Pi, by contrast, is designed to run an operating system called GNU/Linux Raspbian. Hereafter referred to simply as Linux. Unlike Windows or OS X, Linux is open source: it's possible to download the source code for the entire operating system and make whatever changes you desire.

\section{2) TI CC3200 Launchpad Board -}

The TI CC3200 Launchpad consists of Applications Microcontroller, Wi-Fi Network Processor, and PowerManagement subsystems. It uses ARM Cortex M4 Core Processor at $80 \mathrm{MHz}$. It has embedded memory including RAM (256 KB). The dedicated ARM micro-controller also has a network processing subsystem in it. Its features include:
- The board is powered through USB for the Launch Pad and external Booster Pack

- It is operated from 2 AA- batteries

- Standalone development platform provides features such as sensors, LEDs and push-buttons

- On-board antenna and U.FL connector can be selected using a capacitor re-work

- It supports 4 wire JTAG and 2 Wire SWD

- GNU Debugger (GDB) supports over Open On chip debugger (Open OCD)

- Two 20-pin connectors enable compatibility with Booster Packs which have added functions

- Flash memory is updated through USB using Simple Link Programmer

The board can be programmed through Energia IDE over the USB cable. The accessible Wi-Fi used by the board should be proxy less and can be of WPA or WEP type.

\section{Setup}

The motion sensor is connected to a digital in-out pin of Texas board. The board is powered up by external $12 \mathrm{~V}$ battery or $12 \mathrm{~V}$ adapter. The home appliances are connected to mains through relay which in turn is connected to another digital pin of the board. The board is programmed to have access to the local Wi-Fi. The voice call feature of the mobile phone should be enabled.

\section{3) PIR Motion Sensor -}

The PIR (Passive Infra-Red) Sensor is a Pyroelectric device that detects human body motion by measuring changes in the infrared levels emitted by surrounding objects. This motion can be detected by checking for a high signal on a single I/O pin. Incorporating a Fresnel lens and motion detection circuit. High sensitivity and low noise. Output is a standard 5V active low output signal. Module provides an optimized circuit that will detect motion up to 6 meters away Inexpensive and easy to use, The Output can be connected to GPIO pins of Raspberry Pi directly to monitor signal.

The System was implemented on a Raspberry pi development board in Linux environment, which supports SMTP (Simple Mail Transfer Protocol), TCP/IP, HTTP. The web server Flash File System supports dynamically generated files that can include output data from hardware resources. This type of file is called an embedded server page (ESP).

\section{WORKING OF PIR MOTION SENSOR}




\section{International Journal of Innovations in Engineering and Science, www.ijies.net}

Human Beings emits thermal energy of wavelength around 9-10 micro-meter everyday. Pyroelectric or Passive Infrared Sensor (PIR) [6], [7] is an electronic device which is designed to detect this IR wavelength when a human being is in its proximity. To have a wide range for detection a simple lens is used. Sensors may also be calibrated in such a way so as to ignore domestic pets by setting a higher sensitivity threshold, or by ensuring that the floor of the room remains out of focus.

\section{Advantages -}

- This low cost system with minimum requirements takes care of both home security as well as home automation

- This home security system does not use any smart- phone application or any type of user interface instead uses digits from the keypad on the phone, the system is platform independent and hence can be accessed from a wide range of phones with different operating systems.

- To operate home security system the user need not have data connection enabled in his phone. The system runs fine with the launchpad connected to wifi at home/office.

- The optional smart phone application takes care of the fact that the user may also wish to control his home appliances without sensors being triggered.

- To operate home security system the user need not have data connection enabled in his phone. The system runs ne with the launchpad connected to wi at home.

- The optional smart phone application takes care of the fact that the user may also wish to control his home appliances without sensors being triggered [5].

- $\quad$ Since the launchpad sends a voice call to only a particular number which is present in the web API there is no need to worry about security leakage as the system cannot be accessed by any other unauthenticated user. This in turn increases the fidelity of the security system.

- The use of Wi-Fi enabled launchpad in the system enables the system to be controlled from any part of the globe contrary to Blue-tooth controlled or IR remote controlled existing home automation solutions that too without any net connectivity in the phone.

- Since the same set of motion sensors can be deployed for home automation as well as security system the system is simple and inexpensive.

This system does not require the user to manually trigger an alarm but still it provides the user with the advantage of analyzing the situation and then triggering the security alarm remotely from his phone. This idea overcomes the common fault in many exist- ing home security systems which causes unnecessary embarrassment by triggering security alarm due to the systems inability to judge a special situation in which it should not have triggered the alarm.

\section{IV - CONCLUSION \& FUTURE SCOPE}

The sensors positioned on the door informs the home proprietor as quickly as the door is opened through sending a Push notification. The consumer will get this notification irrespective of whether or not the phone is locked or unlocked or even if any different app is opened at the moment. This used to be the fundamental objective of the project, which is the user feels secure and now not worry about any intrusion or break-ins when he is away from home. This setup can additionally be used in business workplaces the place some areas are confined for sure personnel, such a system will right now inform the administrator of any unauthorized personnel trying to access such an area. Therefore the extensibility and applicability of such a system is solely restrained only by way of the imagination. Another essential element of the challenge is the connectivity between the ESP8266 (Wi-Fi module) and the Blynk server. The gadget efficiently related to the Blynk server the usage of the authentication token and the Blynk libraries. As a result, we have been capable to get the notification on our clever phones as soon as there was once any exchange in the repute of the reed module sensor. Also the additional capability to control the alarm remotely is very advisable and can be very beneficial in some unforeseen circumstances. It used to be also found that the Blynk app worked smoothly and carried out all verbal exchange between the hardware and the app very accurately.

The developed device can also be used to in industrial and business purposes such as offices, warehouses and other areas the place some areas are reserved for authorized personnel only or different locations where protection and precautions are of important concerns such as net server room of a large MNC from the place corporate data can be stolen. The gadget can additionally be without difficulty upgraded to add more security aspects such as cameras, action detection sensors, etc. for accelerated safety. The 
Vol. 6, No. 10, 2021, PP. 23-28

\section{International Journal of Innovations in Engineering and Science, www.ijies.net}

gadget can additionally similarly be developed with the aid of adding an RFID scanner so that the authorized users need solely carry a RFID or NFC tag with them on their person. The RFID scanner will work with the aid of scanning the tag wirelessly and if the user is licensed to enter, the alarm device will be disabled for some time so that the user can enter. The captured photograph of the visitor or intruder after face detection can be mailed to the user. The person can further forward the identical photo to the police station if he wishes. Further the gadget may additionally be made greater synchronised by integrating the voice name characteristic inside the equal clever smartphone application through which the consumer can even control his domestic home equipment except any voice name being caused to his phone. Security is of necessary importance in today's world, typical system has attempted to furnish the equal the usage of applied sciences such as microcontroller and up to date variations of the same. The Proposed System provides Security to the house via detecting the presence of any intruder. If any intruder is detected, an alarm is raised and the proprietor and law enforcements are notified through message. The proposed work eliminates the overhead associated with usual machine such as high down time at some point of restore and upkeep and any form of device tampering that an intruder or hacker can do to the system.

\section{REFERENCES}

[1] Govinda K and Sai Krishna Prasad K and Sai ram susheel 2014 Intrusion detection system for smart home using laser rays International Journal for Scientific Research \& $\begin{array}{llll}\text { Development } & \text { (IJSRD) } & 2 & 176-78\end{array}$ Karri V and Daniel Lim J S 2005 Method and Device to Communicate via SMS after a Security Intrusion 1st International Conf. on Sensing Technology Palmerston North New Zealand 21-23.

[2] Jayashri B and Arvind S 2013 Design and Implementation of Security for Smart Home based on GSM technology International Journal of Smart Home 7 201-08

[3] Sowjanya $G$ and Nagaraju $S 2016$ Design and Implementation Of Door Access Control And Security System Based On Iot Inventive Computation Technologies (ICICT), International Conference on Inventive

[4] Cristian C, Ursache A, Popa D O and Florin Pop 2016 Energy efficiency and robustness for IoT: building a smart home security system Faculty of Automatic Control and Computers University Politehnica of Bucharest, Bucharest, Romania 43

[5] Lee C T, Shen T C, Lee W D and Weng $K W 2016$ A novel electronic lock using optical Morse code based on the Internet of Things Proceedings of the IEEE International Conference on Advanced Materials for Science and Engineering eds. Meen, Prior \& Lam

[6] Pooja P, Mitesh P, Vishwa P and Vinit N 2016 Home Automation Using Internet of Things Imperial Journal of Interdisciplinary Research (IJIR) 2 648-51
[7] Anitha A, Paul G and Kumari S 2016 A Cyber defence using Artificial Intelligence International Journal of Pharmacy and Technology 8 25352-57

[8] Anitha A, Kalra S and Shrivastav 2016 A Cyber defence using artificial home automation system using IoT International Journal of Pharmacy and Technology 8 25358-64 